\title{
Not Ready, Not Set. . .Discharge: Patient-Reported Barriers to Discharge Readiness at an Academic Medical Center
}

\author{
James D. Harrison, MPH, PhD*, Ryan S. Greysen, MD, MHS, Ronald Jacolbia, BA, Alice Nguyen, BA, \\ Andrew D. Auerbach, MD, MPH
}

Division of Hospital Medicine, University of California San Francisco, San Francisco, California.

BACKGROUND: Planning for discharge from the hospital should begin early in each patient's stay and focus on the patient's needs.

OBJECTIVE: To determine how often patient-reported barriers to discharge on admission were resolved by discharge and to explore associations between barriers and readmission.

DESIGN, SETTING, AND PARTICIPANTS: A prospective observational study of patients admitted to an academic medical center.

INTERVENTION AND MEASUREMENTS: Patients completed a barriers to discharge survey from the start of hospitalization to discharge. Primary outcomes were the prevalence of discharge barriers, rates of resolution of barriers during hospitalization, and comparisons between barriers identified in admission and discharge surveys.

RESULTS: One hundred sixty-three patients were enrolled, and 68 patients (42\%) completed an admission survey and discharge survey $\leq 48$ hours before discharge. Patients completed on average 1.82 surveys (standard deviation, 1.10; range, 1-8). Total and mean numbers of barriers were highest on the admission survey and decreased until the fourth survey. On average, the total number of barriers to discharge decreased by 0.15 (95\% confidence interval: $0.01-0.30)$ per day $(P=0.047)$. Ninety percent of patients were discharged with at least 1 issue. The 3 most common barriers on the admission and discharge survey remained the same: pain, lack of understanding of recovery plan, and daily-living activities.

CONCLUSIONS: Patient-reported barriers to discharge are prevalent and incompletely addressed. This suggests an opportunity for improved discharge planning and a framework for communication between providers and patients. Journal of Hospital Medicine 2016;11:610-614. (C) 2016 Society of Hospital Medicine
Thirty-six million adults were discharged from US hospitals in 2012, with approximately $45 \%$ from medicine service lines. ${ }^{1,2}$ Discharge planning, a key aspect of care for hospitalized patients, ${ }^{3}$ should involve the development of a plan to enable the patient to be discharged at the appropriate time and with provision of sufficient postdischarge support and services. $^{4}$

Central to the discharge planning process is an assessment of a patient's readiness for discharge. Readiness is often a provider-driven process, based on specific clinical and health system benchmarks. ${ }^{5}$ However, providers' perception of readiness for discharge does not always correlate with patients' selfassessments or objective measures of understanding. ${ }^{6}$ For example, nurses overestimate patients' readiness for discharge compared to patients' own self-report. ${ }^{7}$ As a result, the need to include the patient perspective

*Address for correspondence and reprint requests: James D. Harrison, $\mathrm{PhD}$, Division of Hospital Medicine, University of California San Francisco, 533 Parnassus Avenue, Box 0131, San Francisco, CA 94143; Telephone: 415-502-2008; Fax: 415-514-2094; E-mail: james.harrison@ucsf.edu

Additional Supporting Information may be found in the online version of this article.

Received: September 25, 2015; Revised: March 10, 2016; Accepted: March 14, 2016

2016 Society of Hospital Medicine DOI 10.1002/jhm.2591

Published online in Wiley Online Library (Wileyonlinelibrary.com). is increasingly recognized as an important contributing factor in the discharge planning process. ${ }^{8,9}$

Current approaches to assessing discharge readiness are typically single assessments. However, these assessments do not take into account the complexity of discharge planning or patients' understanding, or their ability to carry out post-acute care tasks. ${ }^{8}$ In addition, few models have included assessments of physical stability and functional ability along with measures such as ability to manage self-care activities at home, coping and social support, or access to health system and community resources. ${ }^{10,11}$

To address these gaps in the existing literature, we carried out a prospective observational study of daily, patient-reported, assessments of discharge readiness to better understand patients' perspectives on issues that could impede the transition to home. Using these data, we then sought to determine the prevalence of patient-reported discharge barriers and the frequency with which they were resolved prior to the day of discharge. We also explored whether problems identified at discharge were associated with 30-day readmission.

\section{METHODS}

\section{Study Design, Setting, and Participants}

We carried out a prospective observational study at the University of California San Francisco (UCSF) Medical Center, a 600-bed tertiary care academic 
hospital in San Francisco, California. The UCSF Committee on Human Research approved this study. We recruited patients between November 2013 and April 2014. Patients were eligible to participate if they were admitted to the General Medicine Service; over 18 years old; English speaking; cognitively able to provide informed consent; and not under contact, droplet, airborne, or radiation isolation. Patients were eligible to participate regardless of where they were admitted from or expected to be discharged (eg, home, skilled nursing facility). Patients were excluded if they were acutely unwell or symptomatic resulting in them being unable to complete the surveys. Caregivers were not able to participate in the study on behalf of patients. We screened daily admission charts for eligibility and approached consecutive patients to consent them into the study on their first or second day of hospitalization. An enrollment tracker was used to documented reasons for patients' exclusion or refusal.

\section{Survey Development}

We adapted an existing and validated Readiness for Hospital Discharge Survey (RHDS) previously used in obstetric, surgical, and medicine patients for our study. ${ }^{10-12}$ This initial list was culled from 23 to 12 items, based on input from patients and physicians. This feedback step also prompted a change in the response scale from a 0 to 10 scale to a simpler "yes," "no," or "I would like to talk with someone about this" scale intended to encourage discussion between patients and providers. After this revision step, we further pretested the survey among physicians and a small set of general medical patients to assess comprehension. Thus, our final question set included 12 items in 4 domains; personal status (ie, pain, mobility), knowledge (ie, medications, problems to watch for, recovery plan), coping ability (ie, emotional support, who to call with problems), and expected support (ie, related to activities and instrumental activities of daily living).

\section{Data Collection}

We collected data from interviews of patients as well as chart abstraction. Trained research assistants approached patients to complete our revised RHDS at admission, which was either on their first or second day of hospitalization. We collected data via an intake admission survey, which asked patients about their readiness for discharge, followed by a daily readiness for discharge survey until the day of discharge. A research assistant read the survey items to patients and recorded responses on a paper version of the survey. We abstracted demographic, clinical, and 30-day readmission information from each participant's electronic medical record.
TABLE 1. Demographic and Clinical Characteristics of Participants $(n=163)^{\star}$

\begin{tabular}{lc}
\hline Mean age, y (SD) & $56.4(17)$ \\
Female gender, no. (\%) & $86(53)$ \\
Race, no. (\%) & \\
Asian & $13(8)$ \\
African American & $27(16)$ \\
White & $96(59)$ \\
Other & $24(25)$ \\
Declined to say & $3(1)$ \\
Married, no. (\%) & $78(48)$ \\
Insurance, no. (\%) & \\
Medicare & $59(36)$ \\
Medicaid & $22(14)$ \\
Private & $73(45)$ \\
Self-pay & $2(1)$ \\
Other & $7(4)$ \\
Patient admitted from, no. (\%) & \\
Home & $118(72)$ \\
Outpatient clinic & $17(10)$ \\
Procedural area & $6(4)$ \\
Another facility & $12(7)$ \\
Other & $9(6)$ \\
Patient discharged to, no. (\%) & \\
Home without services & $107(66)$ \\
Home with services & $40(25)$ \\
Home hospice & $2(1)$ \\
Skilled nursing facility & $8(5)$ \\
Patient deceased & $3(2)$ \\
Other & $3(2)$ \\
\hline
\end{tabular}

NOTE: Abbreviations: SD, standard deviation. *Where data are missing the values do not equal $100 \%$

\section{Analytic Approach}

A barrier to discharge readiness was confirmed when a patient responded "no" to an item (except for presence of catheter and pain or discomfort where "yes" was used) and/or they stated they wanted to talk to someone about the issue. We then used descriptive statistics to summarize patients' responses by survey administration number. Multilevel mixed effect regression was used to investigate any patterns in barriers to discharge over the course of hospitalization. We described the frequency of identified barriers to discharge on the intake admission and final $(\leq 48$ hours of discharge) surveys. McNemar's tests compared the proportion of patients reporting each barrier, and paired $t$ tests the mean number of barriers at these 2 survey time points. We also assessed whether persistent barriers to discharge readiness on the final survey were associated with readmission to our hospital within 30-days using $t$ tests, $\chi^{2}$, or Fisher exact test. Analysis was conducted in SPSS 22.0 (IBM Corp., Armonk, NY) and Stata (StataCorp, College Station, TX).

\section{RESULTS \\ Patients}

There were 2045 patients admitted to the general medicine service during the study period. Medical record screening resulted in 1350 exclusions. Of the 
TABLE 2. Percentage of Eligible Patients Surveyed by Hospitalized Day

\begin{tabular}{lccccccccccc}
\hline & \multicolumn{10}{c}{ Hospital Day } \\
\cline { 2 - 10 } & 1 & 2 & 3 & 4 & 5 & 6 & 7 & 8 & 9 \\
\hline No. of eligible patients hospitalized & 163 & 161 & 138 & 102 & 70 & 50 & 35 & 24 & 19 & 17 \\
No. of patients surveyed & 8 & 124 & 70 & 30 & 22 & 13 & 7 & 6 & 2 & 0 \\
\% of eligible patients surveyed & 4.9 & 77.0 & 50.7 & 29.4 & 31.4 & 26.0 & 20.0 & 25.0 & 10.5 & 0 \\
\hline
\end{tabular}

remaining 695 patients, 113 refused and 419 were further found to be unable to participate. After all exclusions were applied and following direct screening, 163 patients agreed to participate in our study (Table 1). Mean length of stay among our cohort was 5.42 days (standard deviation [SD], 11.49) and the majority of patients were admitted from and discharged to home (Table 1).

\section{Barriers to Discharge Readiness}

Patients completed on average 1.82 surveys (SD 1.10; range, 1-8), and in total 296 surveys were administered. Only $5 \%$ of patients were captured on their admission day, whereas $77 \%$ of patients were surveyed on their second hospital day (Table 2). Between the first and second survey administration, $51 \%$ of patients were lost to follow-up, and then by the third survey administration a further $37 \%$ were lost to follow-up (Table 3). Patients were unable to be reinterviewed most often because they had been (1) discharged, (2) were unavailable or having a procedure at time of recruitment, or (3) became too sick and symptomatic.

In total, over 889 individual barriers to discharge readiness were reported across all surveys. The total and mean numbers of barriers were highest on the admission intake survey, and numbers continued to decrease until the fourth survey. On average, the total number of barriers to discharge patients reported decreased by 0.15 (95\% confidence interval: 0.01 $0.30)$ per day $(P=0.047)$.

\section{Change in Barriers to Discharge}

Sixty-eight patients $(42 \%)$ completed an admission intake survey as well as final survey $\leq 48$ hours before discharge (Table 4). We observed a significant reduction in mean number of barriers reported between admission and discharge surveys (3.19 vs $2.53, P=$ 0.01). Sixty-one patients $(90 \%)$ left the hospital with 1 or more persistent barrier to a safe discharge. However, the 3 most common barriers to discharge readiness on the admission and final survey remained the same: unresolved pain, lack of understanding of plan for recovery, and daily living activities (eg, cooking, cleaning, and shopping). The number of patients with unresolved pain appeared to increase slightly, though this rise was not statistically significant. In contrast, there were significant reductions in patients reporting they were unaware of problems to watch out for postdischarge $(28 \%$ vs $16 \% ; P=0.04)$ or did not understand their recovery plan $(52 \%$ vs $40 \% ; P=0.03)$.

\section{DISCUSSION}

Assessing discharge readiness highlights an opportunity to engage patients directly in their discharge planning process. However, our prospective study of 163 hospitalized adults revealed that unresolved discharge barriers were common; $90 \%$ of patients were discharged with at least 1 issue that might inhibit an effective transition home. The majority of these patients were also discharged home without any support services. In addition, many of the major barriers patients reported-pain, lack of understanding around plans, and ability to provide self-care-were consistent from admission to discharge, suggesting a missed opportunity to address problems present early in a patient's stay.

Some of the issues our patients described, such as pain; lack of understanding of a recovery plan; and functional, social, and environmental vulnerabilities

TABLE 3. Barriers to Discharge Readiness by Survey Number

\begin{tabular}{|c|c|c|c|c|c|c|}
\hline & \multicolumn{6}{|c|}{ Survey No. } \\
\hline & 1 & 2 & 3 & 4 & 5 & $6+$ \\
\hline No. of patients surveyed & 163 & 83 & 31 & 11 & 3 & 5 \\
\hline Total barriers (all patients) & 533 & 235 & 84 & 22 & 7 & 8 \\
\hline No. of barriers per patient, mean (SD) & $3.27(2.35)$ & $2.83(2.11)$ & $2.71(2.49)$ & $2.00(1.73)$ & $2.33(2.51)$ & $1.60(2.30)$ \\
\hline Median no. of barriers per patient & 3.0 & 3.0 & 2.0 & 1.0 & 2.0 & 0 \\
\hline Median hospital day of survey administration & 2.0 & 3.0 & 5.0 & 6.0 & 8.0 & 13.0 \\
\hline Initial admission survey, no. (\%) & $163(100.0)$ & 0 & 0 & 0 & 0 & 0 \\
\hline Follow-up survey, no. (\%) & 0 & $38(45.8)$ & $16(51.6)$ & $4(36.4)$ & 0 & $1(20.0)$ \\
\hline Survey $\leq 48$ hours before discharge, no. (\%) & $59(36.2)$ & $45(54.2)$ & $15(48.4)$ & $7(63.6)$ & $3(100.0)$ & $4(80.0)$ \\
\hline
\end{tabular}


TABLE 4. Barriers Reported by Patients Who Completed More Than One Survey $(n=68)$

\begin{tabular}{lcc}
\hline & \multicolumn{2}{c}{ Survey } \\
\cline { 2 - 3 } Barrier to Discharge & $\begin{array}{c}\text { Admission, } \\
\text { No. }(\%)\end{array}$ & $\begin{array}{c}\text { Final Survey, } \\
\text { No. (\%) }\end{array}$ \\
\hline Catheter is present? & $6(7.2)$ & $6(7.2)$ \\
Not out of bed, sitting in a chair, or walking? & $17(20.5)$ & $13(15.7)$ \\
Pain or discomfort? & $50(60.2)$ & $52(62.7)$ \\
Unable to get to the bathroom for toilet or to shower? & $15(18.1)$ & $12(14.5)$ \\
Unable to self-care without help from others? & $27(32.5)$ & $23(27.7)$ \\
Unable to get your own medications? & $11(13.3)$ & $14(16.9)$ \\
Know what problems to watch for?* & $23(27.7)$ & $13(15.7)$ \\
Know where to call if you had problems? & $10(12.0)$ & $8(9.6)$ \\
Inability for personal care such as bathing, & $8(9.6)$ & $11(13.3)$ \\
$\quad$ toileting, and eating? & & \\
Lack of support for emotional needs? & $16(19.3)$ & $9(10.8)$ \\
Unable to cook, clean, or do shopping? & $33(39.8)$ & $25(30.1)$ \\
Do not understand the overall plan for & $43(51.8)$ & $33(39.8)$ \\
$\quad$ your recovery?* & & \\
\hline
\end{tabular}

NOTE: *Difference between admission and final survey $P<0.05$; all other differences nonsignificant.

that impede recovery, have been described in studies using data collected in the postacute time period. ${ }^{13-15}$ Focus on postacute barriers is likely to be of limited clinical utility to assist in any real-time discharge planning, particularly planning that assesses individual patients' needs and tailors programs and education appropriately. Having said this, consistency between our results and data collected from postdischarge patients again supports broad areas of improvement for health systems.

Persistent gaps in care at discharge may be a result of limited standardization of discharge processes and a lack of engagement in obtaining patient-reported concerns. Lack of a framework for preparing individual patients for discharge has been recognized as a significant obstacle to effective discharge planning. For example, Hesselink et al.'s qualitative study with almost 200 patients and providers across multiple institutions described how lack of a standard approach to providing discharge planning resulted in gaps in information provision. ${ }^{16}$ Similarly, Horwitz et al. described wide variation in discharge practices at a US academic medical center, suggesting lack of a standard approach to identifying patient needs. ${ }^{14}$

Although many transitions of care programs have supported implementation of specific care interventions at a hospital or health system level, there have been surprisingly few studies describing efforts to standardize the assessment of discharge barriers and prospectively engage individual patients. ${ }^{17}$ One emblematic study used stakeholder interviews and process mapping to develop a "readiness report" within their electronic medical record (EMR). ${ }^{17}$ Aggregate data from the EMR including orders and discharge plans were coded, extracted, and summarized into a report. The overall goal of the report was to identify progress toward completion of discharge tasks; however, a limitation was that it did not explicitly include patient self-assessments. Another study by Grimmer et al. describes the development of a patientcentered discharge checklist that incorporated patients and care concerns. ${ }^{18}$ The themes incorporated into this checklist cover many transitional issues; however, outside of the checklist's development, few publications or Web resources describe it in actual use.

Our approach may represent an advance in approaches to engaging patients in discharge planning and preparing patients for leaving the hospital. Although our data do not support efficacy of our daily surveys in terms of improving discharge planning, this initial evaluation provides the framework upon which providers can develop discharge plans that are both standardized in terms of using a structured multidomain communication tool to elicit barriers, as well as patient-centered and patient-directed, by using the information collected in the survey tool to initiate tailored discharge planning earlier in the hospital stay. However, our program points out an important limitation of an entirely patient-initiated program, which is difficulty obtaining truly "daily" assessments. During this study, we had a single research assistant visit patients as frequently as possible during hospitalization, but even daily visits did not yield complete information on all patients. Although this limitation may in part be due to the fact that our study was a focused pilot of an approach we hope to expand, it also represents the complexity of patient experience in the hospital, where patients are often out of their room for tests, are unable to complete a survey because of problematic symptoms, or simply are unwilling or unable to participate in regular surveys.

Our study has a number of limitations. First, the number of patients in our study overall, and the number who completed at least 2 surveys, was relatively small, limiting the generalizability of the study and our ability to determine the true prevalence of unresolved barriers at discharge. In addition, our selection criteria and response rates have limited our sample in that our final group may not be representative of all patients admitted to our medicine service. The broad exclusion of patients who had physical or psychosocial barriers, and those who were acutely unwell and symptomatic, has the potential to introduce selection bias given the excluded populations are those most at risk of readmission. We also acknowledge that some of the issues that patients' are reporting may be chronic ones. However, given the fact that patients feel these issues, even if chronic, are unaddressed or that they want to talk with their doctor about them, is still a very large potential gap in care and patient engagement.

However, despite these limitations, which seem most likely to produce a cohort that is more likely to be able to participate in our survey, and in turn more likely to participate in their care more broadly, we still observed disappointing resolution of discharge 
barriers. In addition, our adapted survey instrument, though based on well-supported conceptual frameworks, ${ }^{19}$ has not been extensively tested outside of our hospital setting. Finally, as a single-center study, our results cannot be generalized to other settings.

Assessing discharge readiness highlights an opportunity to obtain patient self-reported barriers to discharge. This can facilitate discharge planning that targets individual patient needs. This information also emphasizes potentially fruitful opportunities for improved communication and education activities, potentially if these data are fed back to providers in real time, potentially as part of team-based dashboards or the context of interdisciplinary team models.

\section{Acknowledgements}

The authors thank all of the patients who participated in this project, and Yimdriuska Magan "Gigi" for her assistance with chart abstractions. The authors also acknowledge and thank John Boscardin for his statistical and analytic support.

Disclosures: James D. Harrison, and Drs. Ryan S. Greysen and Andrew D. Auerbach contributed to the concept, design, analysis, interpretation of data, drafting of the manuscript, critical revisions to the manuscript, and final approval of manuscript. Ronald Jacolbia and Alice Nguyen contributed to the acquisition of data, drafting and final approval of manuscript and project, and administrative and technical support. Dr. Auerbach was supported by National Heart, Lung, and Blood Institute grant K24 K24HL098372. Dr. Greysen is supported by the National Institutes of Health (NIH), National Institute of Aging (NIA) through the Claude D. Pepper Older Americans Independence Center (P30AG021342 NIH/NIA and K23AG045338-01). The authors have no financial or other conflicts of interest to declare.

\section{References}

1. Weiss AJ, Barrett ML, Steiner CA. Trends and projections in inpatient hospital costs and utilization 2003-2013. HCUP statistical brief \#175. July 2014. Rockville, MD: Agency for Healthcare Research and Quality; 2014.

2. Weiss AJ, Elixhauser A. Overview of hospital stays in the United States 2012. HCUP statistical brief \#180. October 2014. Rockville, MD: Agency for Healthcare Research and Quality; 2014.
3. Joint Commision. The Joint Commission Comprehensive Accreditation Manual for Hospitals. Oak Brook, IL: The Joint Commission; 2015.

4. Alper E, O'Malley TA, Greenwald J. Hospital discharge and readmission. In: Post TW, ed. UpToDate website: Available at: http://www. uptodate.com/contents/hospital-discharge-and-readmission. Accessed August 14, 2015.

5. Anthony MK, Hudson-Barr D. A patient centered model of care for hospital discharge. Clin Nurse Res. 2004;13:117-136.

6. Ubbink DT, Tump E, Koenders JA, Kleiterp S, Goslings JC, Brolmann FE. Which reasons do doctors, nurses and patients have for hospital discharge? A mixed methods study. PLoS One. 2014;9:e91333.

7. Weiss M, Yakusheva O, Bobay K. Nurse and patient perceptions of discharge readiness in relation to postdischarge utilization. Med Care. 2010;48:482-486.

8. Coffey A, McCarthy GM. Older people's perception of their readiness for discharge and postdischarge use of community support and services. Int J Older People Nurs. 2013;8:104-115.

9. Coleman EA, Parry C, Chalmers S, Min SJ. The care transitions intervention: Results of a randomized controlled trial. Arch Intern Med. 2006; $166: 1822-1828$.

10. Weiss ME, Piacentine LB. Psychometric properties of the Readiness for Hospital Discharge Scale. J Nurs Meas. 2006;14:163-180.

11. Weiss ME, Piacentine LB, Lokken L, et al. Perceived readiness for hospital discharge in adult medical-surgical patients. Clin Nurse Spec. 2007;21:31-42.

12. Weiss ME, Costa LL, Yakusheva O, Bobay KL. Validation of patient and nurse short forms of the Readiness for Hospital Discharge Scale and their relationship to return to the hospital. Health Serv Res. 2014; 49:304-317.

13. Greysen SR, Hoi-Cheung D, Garcia V, et al. "Missing Pieces"-functional, social and environmental barriers to recovery for vulnerable older adults transitioning from hospital to home. J Am Geriatr Soc. 2014;62:1556-1561.

14. Horwitz LI, Moriarty JP, Chen C, et al. Quality of discharge practices and patient understanding at an academic medical center. JAMA Intern Med. 2013;173:1715-1722.

15. Graumlich JF, Novotny NL, Aldag JC. Brief scale measuring patient prepardeness for hospital discharge to home: Psychometric properties. J Hosp Med. 2008;3:446-454.

16. Hesselink G, Zegers M, Vernooij-Dassen M, et al. Improving patient discharge and reducing hospital readmission by using intervention mapping. BMC Health Serv Res. 2014;14:389.

17. Tyler A, Boyer A, Martin S, Neiman J, Bakel LA, Brittan M. Development of a discharge readiness report within the electronic health record: a discharge planning tool. J Hosp Med. 2014;9:533-539.

18. Grimmer K, Moss J, Moss J, Kindness H. Incorporating Patient and Carer Concerns in Discharge Plans: The Development of a Practical Patient-Centred Checklist. The Internet Journal of Allied Health Sciences and Practice. 2006; 4: Article 5.

19. Burke RE, Guo R, Prochazka AV, Misky GJ. Identifying keys to success in reducing readmissions using the ideal transitions in care framework. BMC Health Serv Res. 2014;14:423. 